\title{
THz spectroscopy of the atmosphere for climatology and meteorology applications
}

\author{
Peter Hargrave*a, Stafford Withington ${ }^{\mathrm{b}}$, Stefan A. Buehler ${ }^{\mathrm{c}}$, Lukas Kluft ${ }^{\mathrm{c}}$, Ben Flatman ${ }^{\mathrm{a}}$, Prateek \\ Kumar Dongre ${ }^{\mathrm{a}}$ \\ ${ }^{\text {a} C a r d i f f ~ U n i v e r s i t y, ~ S c h o o l ~ o f ~ P h y s i c s ~ \& ~ A s t r o n o m y, ~ Q u e e n s ~ B u i l d i n g s, ~ C a r d i f f ~ C F 24 ~ 3 A A ~}$ \\ ${ }^{\mathrm{b}}$ University of Cambridge, Cavendish Laboratory, J.J. Thomson Avenue \\ Cambridge CB3 OHE \\ 'Universität Hamburg, Geowissenschaften, Meteorologisches Institut, Bundesstr. 55, 20146 \\ Hamburg, Germany
}

\begin{abstract}
We present a new satellite-based instrument concept that will enable global measurements of atmospheric temperature and humidity profiles with unprecedented resolution and accuracy, compared to currently planned missions. It will also provide global measurements of essential climate variables related to ice clouds that will better constrain global climate models. The instrument is enabled by the use of superconducting detectors coupled to superconducting filterbank spectrometers, operating between $50 \mathrm{GHz}$ and $850 \mathrm{GHz}$. We present the science drivers, the current instrument concept and status, and predicted performance.
\end{abstract}

Keywords: hyperspectral, microwave, temperature, humidity, sounder, superconducting, transition edge sensor, kinetic inductance detector

\section{INTRODUCTION}

Over the last two decades, there have been quite significant improvements in the efficacy of numerical weather prediction (NWP) models, thanks to the increased usage of satellite observations in data assimilation systems. Reduction in errors for accurate weather forecasting requires improved Global measurements of atmospheric temperature and humidity. This requires instruments with high sensitivity, low radiometric noise, and high vertical resolution (high spectral resolution and good spectral coverage).

For climatology studies, a deeper understanding of how clouds will respond to a warming climate is one of the outstanding challenges. Uncertainties in response of clouds, and particularly shallow clouds, have been identified as the dominant source of the discrepancy in model estimates of equilibrium climate sensitivity. In particular, the role of ice clouds in the global climate system is a major source of uncertainty in Global circulation models. The role played by fluctuations in water vapour and the coupling of water vapour and atmospheric circulations is critical to our understanding of climate feedback processes. Improved accuracy and vertical resolution profiling of water vapour in the lower troposphere is required to reduce uncertainties in cloud-climate feedbacks and convection initiation.

For atmospheric temperature sounding, major contributors to the reduction of forecast errors are the Advanced Microwave Sounding Unit-A (AMSU-A), on board several platforms [1], and the Infrared Atmospheric Sounding Interferometer (IASI) hyperspectral instrument on board the Meteorological Operational (MetOp) satellites [2-8]. For water vapour retrieval, currently one of the most informative satellite radiometers for humidity soundings is the Advanced Microwave Sounding Unit-B/Microwave Humidity Sounder (AMSU-B/MHS) [1].

However, all of these instruments have drawbacks. Retrievals from infrared hyperspectral instruments such as IASI are considered to be of high quality. But these observations are restricted to clear sky conditions only. The microwave instruments can see down into clouds, and therefore provide important information. But the vertical resolution is very poor, due to the very limited $(<10)$ number of sounding channels. The radiometric noise in these channels is also a limitation on performance. 
We introduce in this paper a new instrument concept, one that that has potentially better performance (in terms of retrieval accuracy) than the infrared (IR) instruments, and is not restricted to only clear sky conditions. The instrument is enabled by on-chip superconducting resonators, coupled to superconducting detectors. The proposed device will couple the incoming signal through a broad-band antenna, which feeds into a co-planar waveguide or microstrip. The signal is then passed through a filterbank in the form of an array of superconducting resonators. The signal coupled into each resonator is read out with either a very sensitive transition edge superconducting (TES) detector, or a Kinetic Inductance Detector (KID). This technology should allow the incoming signal to be split into up to 1,000 narrow-band spectral channels. This new solution will allow full access to the $50-60 \mathrm{GHz}$ atmospheric oxygen lines, which are critical for high-quality atmospheric temperature retrievals, with the potential for continuous spectral coverage up to $\sim 1 \mathrm{THz}$.

This technology will enable a new generation of high-accuracy temperature and humidity sounding instruments, to enable improved weather forecasting ability. At the same time, the ability to cover much higher frequencies with the same instrument will provide critical data for climate modellers to better constrain global climate models.

\section{ATMOSPHERIC SOUNDING FOR NUMERICAL WEATHER PREDICTION}

For atmospheric temperature sounding, the AMSU-A instrument has a reasonably low radiometric noise and is rather insensitive to non-precipitating clouds, but is limited to 10 sounding channels around the oxygen band at $60 \mathrm{GHz}$ for retrieval of atmospheric temperature profiles. On the other hand, the IASI instrument has 8461 channels in the infrared spectrum (from 4-15 $\mu \mathrm{m}$ ), but cloudy pixels have to be precisely identified and rejected in order to avoid any contamination when retrieving temperature information. Moreover, among the whole set of available channels only about 100 are currently assimilated in NWP models, for both practical and scientific reasons [9].

For water vapour retrieval, the AMSU-B/MHS instruments are widely used in satellite data assimilation systems for numerical weather prediction, even though the limited number of spectral channels provides very poor vertical resolution [10-13].

A recent European Space Agency (ESA) funded study by Prigent et al. [14-16] compared the predicted retrieval performance of a future hyperspectral microwave instrument to the predicted performance of the microwave (MW) instruments selected to fly on board the next generation of European operational meteorological satellites (MetOp-SG). For clear sky conditions, the reduction in retrieval uncertainty, compared to a priori information, goes from $2 \%$ to $10 \%$, depending on the atmospheric layers, and is more than twice as good as what will be achieved with MetOp-SG. Improvements compared to a priori for humidity sounding can reach $30 \%$, a significant benefit as compared to MetOp$\mathrm{SG}$ results, especially up to $250 \mathrm{hPa}$. Maximum impact on the results is provided by the hyper-spectral information on the $\mathrm{O}_{2}$ band around $60 \mathrm{GHz}$. Mahouf et al. [15] found that increasing the number of spectral bands around $55 \mathrm{GHz}$ by a factor of three increases the information content on temperature by a factor of around 1.5. They found a clear benefit, in terms of information content, in increasing the number of spectral channels around the $55 \mathrm{GHz} \mathrm{O}_{2}$ and $183 \mathrm{GHz}_{2} \mathrm{O}$ absorption lines, compared to current and future instruments. Higher frequencies will bring information on cloud ice and solid precipitation.

The addition of MW hyperspectral observations will significantly enhance observations and retrieval capabilities. Firstly, if both IR and MW instruments observe the same geophysical variables (i.e., temperature and water vapour profiles), using multiple observations will bring more information to the retrieval. Secondly, since IR and MW observation noises are independent, the so-called "de-noising synergy" stipulates that using both IR and MW observations will reduce this noise and improve the retrievals. Aires et al. $(2011)[17,18]$ showed that the IASI retrievals are significantly improved when AMSU-A and MHS observations are added to the retrieval. With better MW observations from a hyperspectral MW instrument, we expect to improve even further the synergy with the IR hyper-spectral observations from IASI or the next generation of IASI instruments.

A very important aspect in favour of a hyperspectral MW instrument for NWP applications is the fact that the microwave spectrum is less affected by clouds than the infrared one. The operational assimilation of clear sky infrared radiances in NWP models require stringent quality controls in order to exclude data that could be contaminated by clouds in the field of view of the instrument. Such quality controls are less drastic in the microwave allowing more radiances to be assimilated. This would be the case for a hyperspectral microwave instrument (as proposed here) compared to an instrument like IASI. This could potentially greatly increase the number of satellite observations assimilated in the NWP centres. 


\section{CLIMATOLOGY APPLICATIONS}

Cloud ice and water vapour are the two components of the hydrological cycle in the upper troposphere, and both are currently poorly measured. The hydrological cycle is the most important subsystem of the climate system for life on the planet, and hence its understanding is an important scientific goal. Great progress has already been made towards this goal, except in the upper troposphere, where our knowledge is still very limited.

Ice clouds play an important role in the energy budget of the atmosphere. They are at high altitudes, absorb longwave radiation from below and, as they are cold, emit little infrared radiation. This greenhouse effect warms the Earthatmosphere system. On the other hand, cirrus clouds reflect incoming solar short wave radiation and hence cool the Earth-atmosphere system. The net effect is crucial for the atmosphere, but will depend highly on the cloud's horizontal extent, vertical position, ice water content, and ice particle microphysical properties, which all influence the cloud's optical thickness. Thus, the special properties of cirrus clouds have a strong impact on radiative exchanges. Furthermore, cirrus clouds affect the atmospheric energy budget by releasing latent heat during the depositional growth of ice particles and by absorbing heat upon their sublimation. Eventually ice particles, if large enough, settle through the atmosphere and may enhance precipitation generation in lower clouds by the seeder-feeder mechanism.

The importance of clouds in weather and climate processes has been recognized through a number of observational and modelling studies and is emphasized in the third Report of the Intergovernmental Panel on Climate Change (IPCC) [19]. All general circulation models (GCMs) for weather prediction and climate simulation now forecast the vertical distribution of condensate [20,21]. Most models contain the liquid and ice water content (LWC and IWC) as prognostic variables, and forecast their value at each model time step and grid-point from separate water mass continuity equations [see e.g., [22]]. However, the models contain considerable uncertainties and oversimplifications, largely introduced by the assumptions on ice sedimentation velocities and by the treatment of advection from one time step to the next. Thus, recent sensitivity studies, like the ones by Wilson [2000] [23] and Reinhardt and Wacker [2004] [24], find that LWC and IWC vary considerably depending on the assumptions made. To illustrate this, Figure 1 (left plot) shows a climatology for zonal annual mean ice water path (IWP) for different climate models from the IPCC AR4 model data archive. The IWP is the vertically integrated IWC. It varies by up to an order of magnitude between the different models. The discrepancies in the model predicted IWP response to a $\mathrm{CO}_{2}$ doubling (right plot of Figure 1) are even more striking. Even the sign of the response is uncertain. Some models predict an increase of cloud ice at latitudes where others predict a decrease.

These discrepancies in IWP arise because the different models make different assumptions on ice particle properties and on how cloud ice particles are converted to precipitation. Most important in this respect are assumptions on ice particle size $(D)$, mass, and cross-sectional area, because these properties directly influence the particle fall velocities [25]. To improve climate prediction it is necessary to validate the IWC fields of GCMs and resolve the discrepancies. For this purpose, direct global measurements of IWC or its vertical integral IWP are required, at a resolution compatible with the GCMs and fine enough to resolve typical cirrus features. Global measurements of $D$ are needed to constrain the GCMs assumptions on ice particle size, and hence ice particle fall velocity.

Submillimetre (sub-mm) radiometric measurements from satellite or aircraft in the frequency range of 300-1000 GHz have been proposed to investigate cirrus clouds [26, 27]. And the ice cloud instrument (ICI) to be launched on board MetOp-SG will go some way to addressing this need. But the filterbank spectrometer solution presented in this work will provide constraints on these essential climate variables with unprecedented accuracy. 

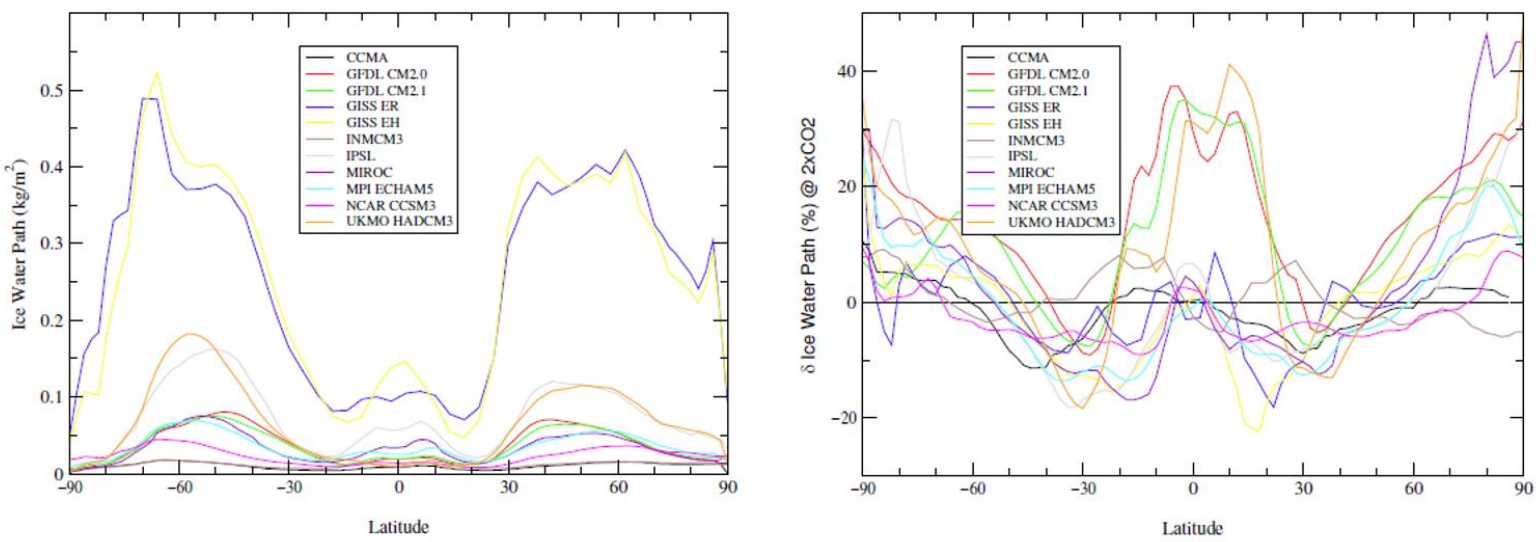

Figure 1. Left: The climatology of zonal annual mean IWP from various climate models in the IPCC AR4 archive. Right: Model predictions of the relative change in zonal annual mean IWP after a $\mathrm{CO}_{2}$ doubling. Note the large discrepancies between different models. Figure from John \& Soden, 2006 [28]

\section{SATELLITE MEASUREMENTS}

For meteorological applications, daily global coverage is essential, with as short a delay in receiving \& processing data as possible (typically between 1 and 4 hours). The horizontal resolution requirement depends strongly on application. For instance, the maximum requirement is $50 \mathrm{~km}$ for current global NWP, $10 \mathrm{~km}$ for future NWP and climate applications, and $1 \mathrm{~km}$ for nowcasting and future regional applications.

For climatological applications, daily global coverage is highly desirable.

Good spatial coverage combined with high spatial resolution intrinsically limits the integration time per ground pixel, and therefore requires high-sensitivity detection with low noise, particularly if the instrument is scanned.

Simultaneous IR data are useful to sample the smallest ice particles and thin cirrus clouds, and for the provision of clearsky temperature and humidity data. In principle, there are two options to achieve this, a dedicated IR radiometer on board the satellite, or a tandem flight with an existing satellite with IR channels. We propose the second option, and fly in tandem with MetOp. The IR data will be provided by the AVHRR/3 and IASI instruments on MetOp. The co-registration of the data will be done in the data processing chain. The high resolution AVHRR $/ 3$ data $(1.1 \mathrm{~km}$ resolution at nadir) can relatively easily be mapped to the larger pixels of a MW instrument and will additionally provide an estimate of the subpixel cloud inhomogeneity. The mapping of the coarser resolution IASI data (12 km resolution at nadir) to the MW pixels will be more difficult, but will provide added value for scientific studies of not too inhomogeneous cloud types, such as large scale cirrus.

The combination with MetOp has the important additional advantage that the full meteorological sensor suite of MetOp can be used to provide complementary data for full sky temperature, humidity and IWP retrieval. Particularly useful will be the humidity and temperature data from MHS and IASI, as well as data on cloud top altitude and cover from AVHRR/3.

The cloud scene at $5 \mathrm{~km}$ resolution should not change significantly between the MetOp and the MW measurements, leading to a threshold time difference between the two measurements of 10 minutes and a target time difference of 1 minute.

Scattering by ice particles introduces polarization effects. It is therefore necessary to observe at a fixed Earth incidence angle and with fixed polarization characteristics. These requirements can be met by a conical scanner. Low incidence angles are desirable in order to achieve a low sounding altitude, and hence to capture a large fraction of the total ice mass and water vapour column. But larger incidence angles are desirable in order to achieve a wide swath, and move towards global coverage in a shorter time interval. This was studied in detail by Jarret et al. [29], who concluded that viewing angles should not exceed $60^{\circ}$ from nadir. The exact value is not very critical, since opacity changes only slowly for moderate viewing angles. A good compromise is an Earth incidence angle of approximately $53^{\circ}$, which is also consistent with other conically scanning satellite instruments. 


\section{FILTERBANK SPECTROMETER DEVELOPMENT}

Studies for hypothetical hyperspectral microwave sounders [14-16] have shown that adding more channels particularly around the $\mathrm{O}_{2}(60 \mathrm{GHz})$ and $\mathrm{H}_{2} \mathrm{O}(183 \mathrm{GHz})$ absorption lines significantly enhances the retrieval capabilities for high accuracy temperature and humidity sounding. Additionally, for accurate retrieval of IWP, it is important that a significant part of the ice particle size distribution is sampled.

The exact channel set to be implemented needs to be fully evaluated by a dedicated study, in order to derive the optimal channel set for maximum information content retrieval, with minimum channel redundancy. However, as a first iteration, we propose to implement an instrument with frequency coverage in the range $50-800 \mathrm{GHz}$ with up to 1000 strategically-placed channels. Each spectral channel will have a bandwidth $(v / \Delta v) \sim 1000(0.1 \%)$.

\subsection{Filterbanks based on kinetic inductance detectors (KIDs)}

On-chip superconducting filterbanks with KID detecting elements are being developed in the DESHIMA [30] and SUPERSPEC [31] projects. Prototype devices have already demonstrated spectral resolutions of around $800(v / \Delta v)$, and near-background-limited performance. These on-chip filterbanks use a series of superconducting microresonators instead of, for instance, an optical grating to divide the incoming signal into separate wavelength bins. The advantage of using KIDs is that they are relatively simple to fabricate, and can be multiplexed to a high ratio, with up to 1000 elements being readout on a single feedline [32]. Note that response below $140 \mathrm{GHz}$ is currently not possible using KID detectors based on Aluminium films, due to the photon energy required to break a Cooper pair in the superconducting film.

\subsection{Filterbanks based on transition edge sensors (TES)}

We are currently developing a low-noise filterbank chip spectrometer based on transition edge sensors [33-37]. We believe that it more prudent, certainly at long wavelengths, to use TESs as the primary sensing elements. No other group has adopted this approach, which is far more secure, certainly at longer wavelengths, than using KIDs. Indeed, TESs continue to be the preferred technology for many areas of observational astrophysics. Although KIDs offer great promise, particularly with respect to multiplexed arrays having hundreds of thousands of pixels, TESs are still seen as the most reliable way of delivering low-noise, high dynamic range, well-calibrated observations. In those cases where "only" hundreds or thousands of pixels are needed, TES-based instruments continue to be proposed and built. Importantly for this application, TESs are eminently suitable for realizing instruments working at frequencies lower than about $140 \mathrm{GHz}$ whereas KIDs are not.

This is a critical advantage - using TESs will allow us access to the very important 50-60 GHz temperature sounding region, something not yet possible with KID technology.

TESs can be designed for both high and low background observations with confidence that designs will perform according to the specifications laid down. They have demonstrated NEPs that are much lower than KIDs, have higher saturation powers, and are more adaptable to background loading effects. TESs can also be more easily coupled to RF microstrip feed lines. With the advent of Frequency Domain Multiplexing (FDM), which is the baseline for the FIR TES instrument SAFARI [37-39] on the SPICA space telescope, and the X-ray TES instrument IXFU on the ATHENA space telescope [40], and with the realization of SQUID multiplexers based on superconducting resonators, very large arrays (many thousands of pixels) can be realized.

Our single-chip filterbank spectrometer will comprise an array of TES sensors connected to an array of thin-film RF filters, which in turn are connected to a single incoming microstrip transmission line. The whole spectrometer can be lithographically fabricated using superconducting films, giving extraordinary performance and functionality. A complete spectrometer having many tens of channels could be fabricated on a single Si chip measuring just $2 \times 2 \mathrm{~cm}$. Although our demonstrator will target the frequency range 50-60 GHz, the underlying technology will be suitable for frequencies ranging from a few tens of $\mathrm{GHz}$ up to many hundreds of $\mathrm{GHz}$, and for spectrometers having several hundred spectral channels. Each TES would comprise a MoAu bilayer on a $200 \mathrm{~nm}$ SiNx membrane, with near ballistic phonon transport along lithographically patterned legs [41] (Figure 2). RF power would be brought onto each TES using a thin-film superconducting microstrip transmission line, which runs along one of the supporting legs, and then deposited in a tiny $\mathrm{AuCu}$ load on the membrane. We could also use $\mathrm{Nb}$ and NbN TESs for higher temperature operation. 


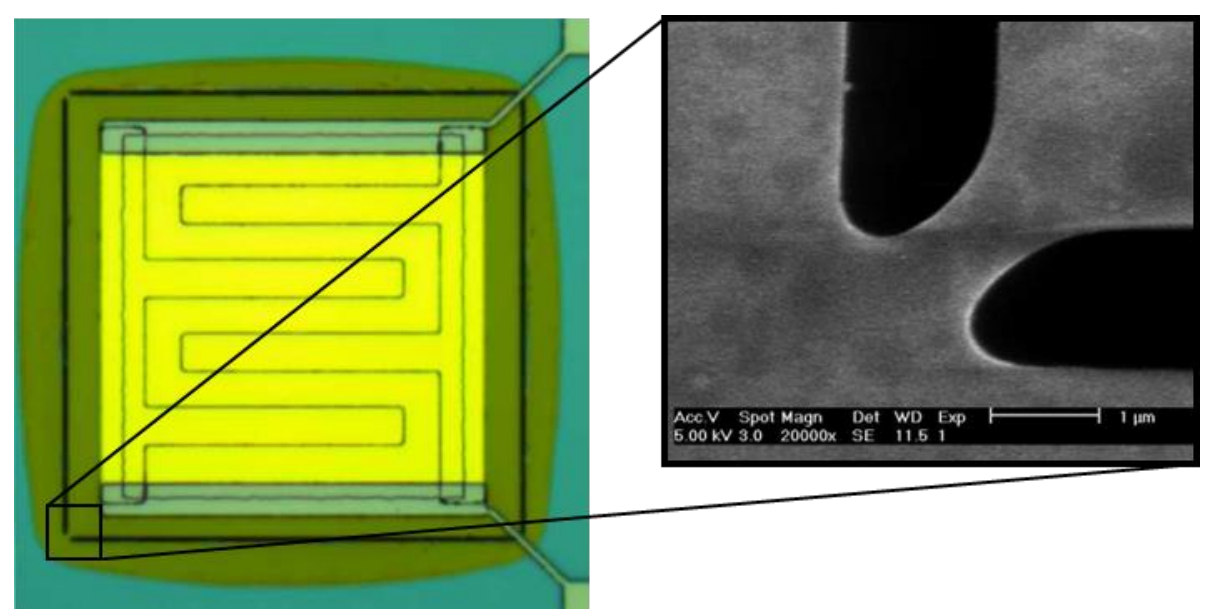

Figure 2. (Left) A photograph of a few-mode ballistic TES; (right) a Scanning Electron Microscopy (SEM) image of one of the dielectric support legs, with a $1 \mu \mathrm{m}$ rule for scale

A high performance filterbank spectrometer will be realized, where the individual RF filters can be patterned using superconducting microstrip technology, where a typical microstrip is just $2 \mu \mathrm{m}$ wide, and the $\mathrm{SiO}_{2}$ dielectric just $200 \mathrm{~nm}$ thick. A key feature of superconducting transmission lines is that because of the kinetic inductance effect, the wavelength of radiation is substantially reduced, leading to physically small distributed RF components. Also, at these wavelengths, the RF components will be essentially lossless, leading to near-ideal filter characteristics.

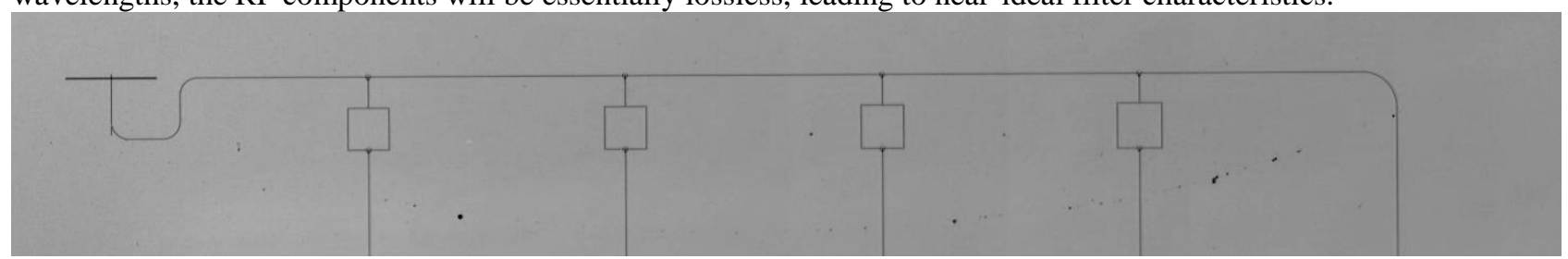

Figure 3 Prototype 4 channel $100 \mathrm{GHz}$ filterbank spectrometer based on superconducting microstrip lines. A slot antenna and total power channel can also be seen.

A key feature of our approach is that we can realise miniature on-chip filters having almost any passband characteristic, and place the centre frequencies anywhere over the band of interest. Thus the individual spectrometer channels can target particular bands without any harmonic, or other, relationship between them. It would also be possible to fabricate a total power detector on the same chip for calibration purposes. We commonly fabricate a miniature (tens of microns in size) chip resistor on each TES membrane in order to provide an absolute calibration against a DC power source. This means that each TES can be absolutely calibrated against a DC source, which would be invaluable for precise power measurements. KIDs cannot be calibrated in this way.

Each device will be read out using a superconducting SQUID, which is mounted in the same box as the spectrometer chip, but in a different sealed cavity. For large numbers of spectral channels, Frequency Domain Multiplexing (FDM) will be used.

\section{INSTRUMENT CONCEPT}

The instrument concept we propose is a conically-scanning instrument with a scan system and antenna similar to that under development for MWI [42-45], in order to minimise development effort and risk. MWI is a conically scanning radiometer, with a $75 \mathrm{~cm}$ diameter primary aperture. The MWI instrument (antenna and receivers) rotates continuously about an axis parallel to the local spacecraft vertical, and the antenna system will view the Earth scene with a nearly constant incidence angle of about $53^{\circ}$. The proposed mechanical layout of MWI is shown in Figure 4. Although the antenna and receiver assembly rotates continuously, a fixed rod through the scan assembly emerges above the rotating drum upper plane to support two calibration targets that are viewed by the receiver for a portion of each scan. The cold calibration target is oriented towards deep space, in the anti-sun direction. We propose to use elements of the MWI 
system, but with changes to the optics, the receiver system, and the requirement for active cooling. We discuss these aspects below.

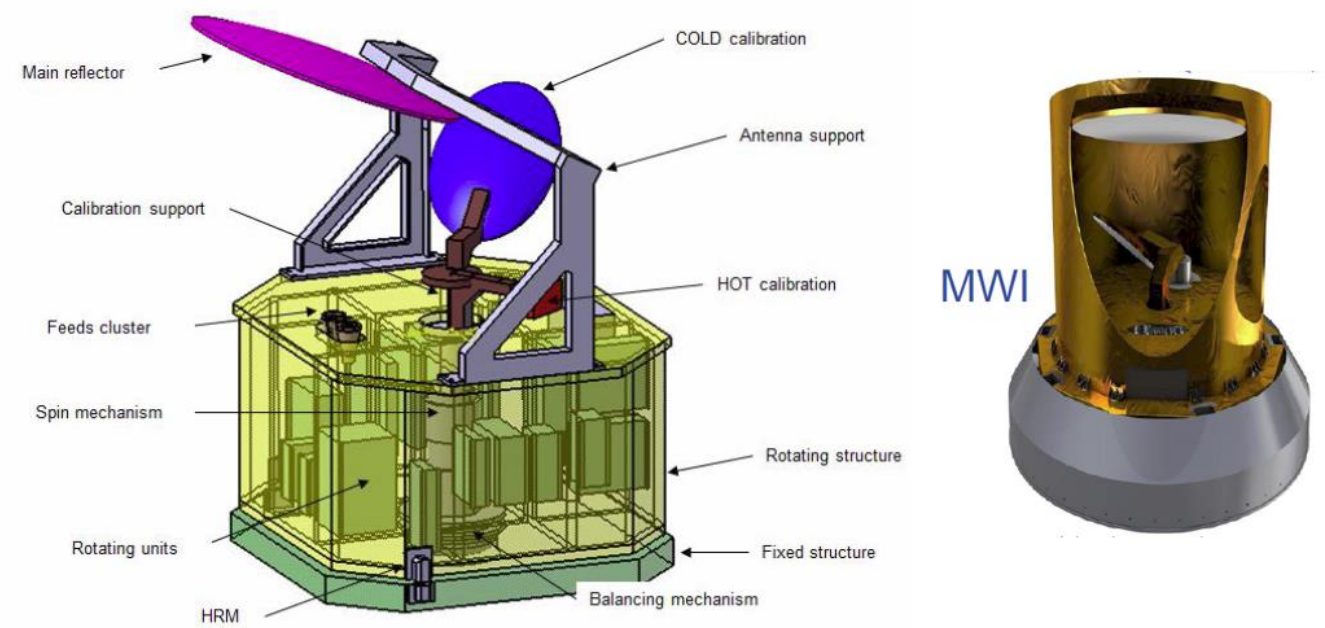

Figure 4 Schematic of the microwave imager for MetOp-SG

\subsection{Receiver system}

The key difference from MWI is the replacement of the heterodyne receivers with our ultrasensitive on-chip spectrometers. This will enable near-photon-noise limited detection capability spanning 50-800 GHz, with channel resolutions of around $1000(v / \Delta v)$. This concept has several major advantages over equivalent spectroscopy systems:

- An order of magnitude increase in the number of detectors (and therefore spectral channels) compared to other sub-mm spectroscopy instruments developed for astronomy and Earth observation applications

- Large instantaneous bandwidth, and the freedom to sample only discrete bands within the spectrum

- Dramatic reduction in overall instrument volume and complexity, as one instrument could exceed the capabilities and coverage of all three microwave/sub-mm instruments (MWS, MWI, ICI) to be flown on MetOp-SG

The typical bandwidth of a filterbank would in practice be around 1 octave. We would envisage using closely-spaced lens-coupled antennas to feed filterbanks covering adjacent spectral bands. E.g. 50-100, 100-200, 200-400, 400-800 GHz. Each antenna and filterbank combination could be arranged similarly to that proposed for DESHIMA [30], as illustrated in Figure 5. This would allow for three or four antenna/spectrometer banks to cover the complete frequency range at one polarization, and the other three/four antenna/spectrometer banks to cover the orthogonal polarization. This arrangement also adds redundancy, if one were to sacrifice polarization capability. Note that it may prove beneficial to replace the lenses with broadband feedhorns. This would be investigated as part of a detailed instrument design study. 


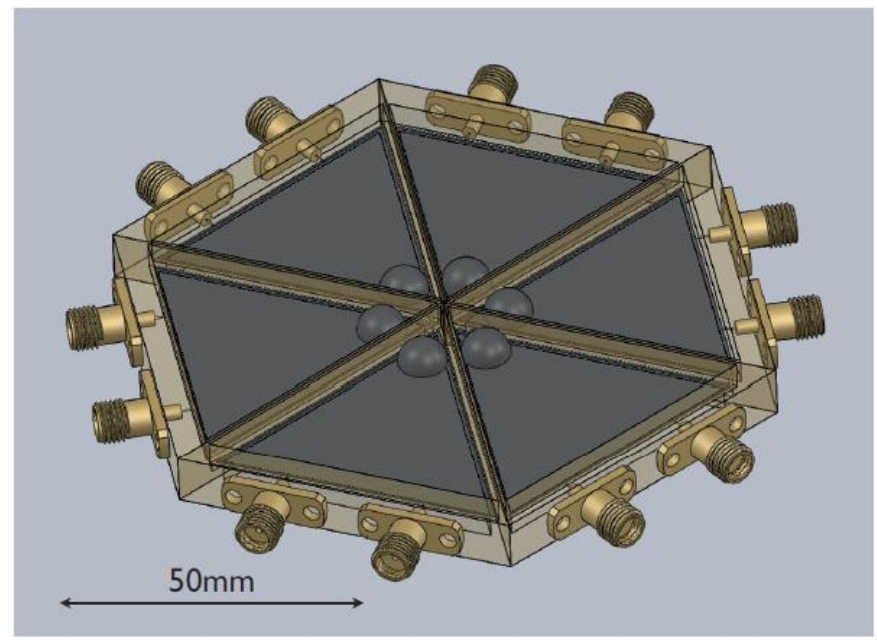

Figure 5. Possible arrangement of antennas and filterbanks. The antennae are situated under the lenses (dome structures), and are at the focus of the parabolic scan mirror. Figure taken from Endo et al. [30].

\subsection{Optics \& scan system}

The lenses (or feedhorns) are placed at the focus of a parabolic primary mirror. Alternatively, the primary focus could be reimaged by cold optics, which would provide a useful cold stop for control of beam sidelobes. A possible implementation of the optical system would be as indicated in Figure 6.

The parabolic primary mirror views a planar scan mirror to implement the required conical scan pattern. Attached to the scan mirror driveshaft could be additional mirrors to intercept the detector beam prior to the cold optics to direct it to view calibration loads on a portion of each scan. We could also consider simply rotating the primary parabolic mirror to implement the scanning, and dispensing with the planar scan mirror. The optical and scan system requires further system-level study, particularly in regard to polarization effects and calibration implementation, to derive the optimal solution.

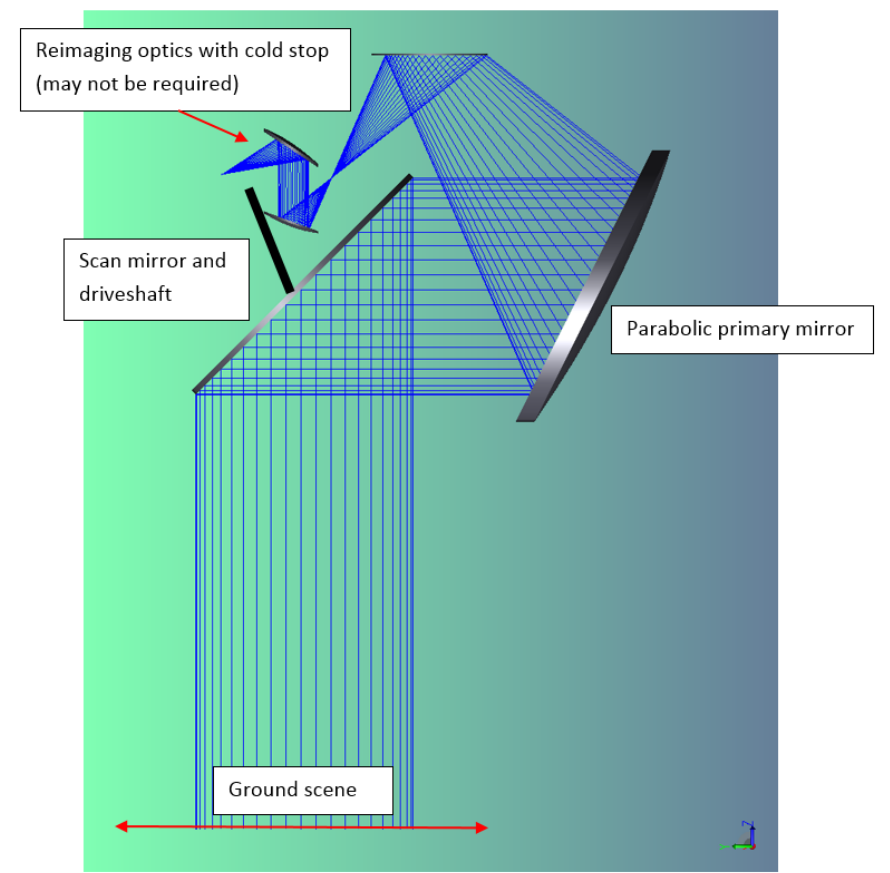

Figure 6. Schematic of possible optical system 


\subsection{Cooling system and thermal considerations}

The focal plane would need to be cooled to $\sim 300 \mathrm{mK}$, with an operational design lifetime goal of around 7 years. This requires high reliability mechanical cryocoolers, attached to a ${ }^{3} \mathrm{He}$ sorption cooling system. The ${ }^{3} \mathrm{He}$ system would be based upon proven heritage from Herschel PACS [46] and SPIRE [47] instruments, with the possibility of continuous $300 \mathrm{mK}$ cooling, recently developed by Cardiff University, in collaboration with Chase Research.

The Planck [48] 4K cooler was developed by the Rutherford Appleton Laboratory as part of the cryogenic chain for the High Frequency Instrument on the Planck spacecraft, which was launched in May 2009. The cooler is based upon the Joule-Thomson (JT) effect, in which a fluid is cooled by adiabatic expansion through a throttled orifice, to fill a small liquid He reservoir on the $4 \mathrm{~K}$ plate. The Planck cooler is a closed cycle system, the working fluid is $\mathrm{He}^{4}$ and, as part of the complex Planck cryogenic chain, the $4 \mathrm{~K}$ cooler made use of two external thermal interfaces to pre-cool the He gas to well below its inversion temperature; there was a $50 \mathrm{~K}$ stage provided by passive cooling from ' $\mathrm{v}$-groove' radiators and further pre-cooling at $17.5 \mathrm{~K}$ is provided by a Hydrogen sorption cooler. The $4 \mathrm{~K} \mathrm{JT}$ cooler provided $20 \mathrm{~mW}$ of heat lift at the $4 \mathrm{~K}$ plate for the continuation of the Planck chain to a further JT and $\mathrm{He}^{3} / \mathrm{He}^{4}$ dilution refrigerator to ultimately reach $0.1 \mathrm{~K}$ at the detectors.

On our proposed instrument, the situation is slightly different in that the $50 \mathrm{~K}$ passive pre-cooling will be replaced by a passive radiator operating at $80 \mathrm{~K}$. This means that there will need to be a re-design of the cooler to accommodate the higher first stage operating temperature. The radiator design is a critical element of such an instrument, and requires detailed study. In such a proposed orbit, the anti-Sun direction is still exposed to significant infrared load from the Earth. The Planck JT compressors were supplied by EADS Astrium, based upon a licensed design from RAL, the compressors are first generation linear motor reciprocating mechanisms originally developed for Stirling cycle coolers which have now amassed around 15 years of in-orbit operational life between 24 mechanisms.

The $4 \mathrm{~K}$ cooler requires pre-cooling to temperatures below the inversion temperature for helium (about $27 \mathrm{~K}$ ). In practice, the lower the pre-cooling temperature (within limits), the better the cooling power. The RAL two-stage $15 \mathrm{~K}$ cooler has been in development for many years and is now able to deliver around $250 \mathrm{~mW}$ at $15.4 \mathrm{~K}$ and a no load base temperature of $9.8 \mathrm{~K}$. The compressor input power under these conditions would be around $135 \mathrm{~W}$. This could provide the basis of an excellent pre-cooler for our instrument.

\section{PREDICTED PERFORMANCE AND PRELIMINARY RETRIEVAL SIMULATIONS}

Preliminary temperature and humidity retrieval simulations have been carried out for clear-sky conditions. Simulations were run to compare the performance of an idealised IASI-type hyperspectral IR sensor to a proposed hyperspectral KID-based microwave sensor. The IR sensor was set up with 8534 channels in the range 19-83 THz, with channel sensitivities (NE $\Delta \mathrm{T})$ in the range $0.1-0.2 \mathrm{~K}$. The microwave/sub-mm sensor was set up with 1000 channels in the range $100-850 \mathrm{GHz}$, with NE $\Delta \mathrm{T}$ values in the range $0.007-0.024 \mathrm{~K}$.

Note that these simulations were run for a KID-based instrument, where access to the important frequencies below $100 \mathrm{GHz}$ was not possible. The technology we propose here will allow access to the critical 50-60GHz $\mathrm{O}_{2}$ temperature sounding lines, which should significantly enhance capability.

Clear sky radiances were calculated using ARTS (Atmospheric Radiative Transfer Simulator) [49]. Comparing the IR and MW spectra (Figure 7), the MW spectrum is much "cleaner". Although IASI has over 8000 channels, there are many more absorption lines, and we get several lines in each spectral bin. In the MW, as well as having a cleaner brightness temperature spectrum, the overall opacity is lower, allowing us to probe deep into clouds. Also, looking at the humidity Jacobian in particular, the brightness temperature is very sensitive to water vapour content and altitude. 


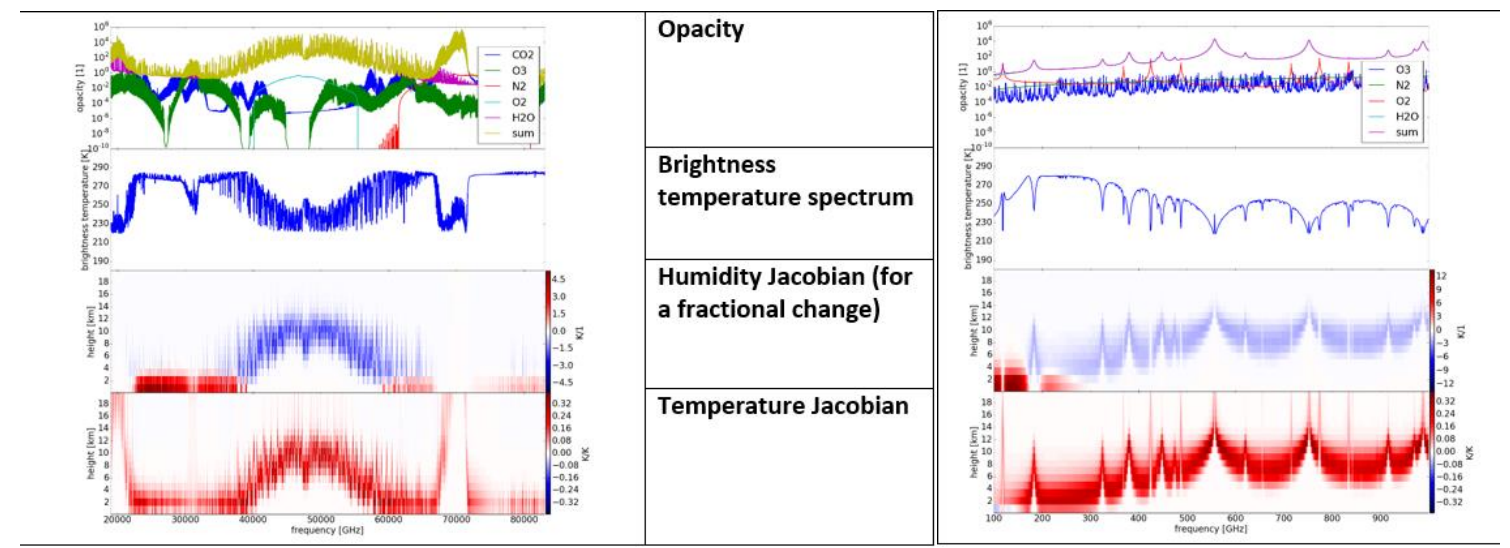

Figure 7. Clear-sky radiative transfer simulations of the atmosphere in the infrared (left) and microwave/sub-mm (right) regions.

Retrieval simulation results are presented in Figure 8 to Figure 10. Figure 8 shows the predicted retrieval error for water vapour as a function of altitude.

IR

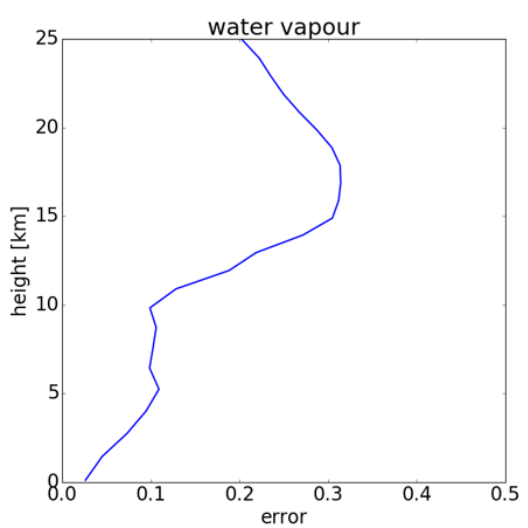

KID Microwave

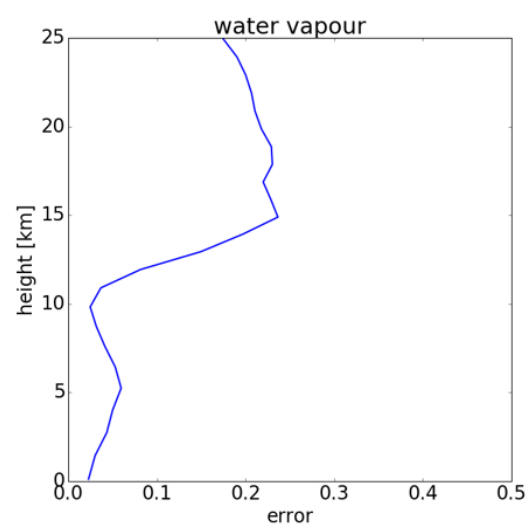

Figure 8. Predicted water vapour retrieval error for IR and KID-based microwave instruments. Absolute error in $\ln \left(\mathrm{VMR}\left(\mathrm{H}_{2} \mathrm{O}\right)\right)$ coordinates -0.2 corresponds roughly to $20 \%$ relative error.

It can be seen that the performance of the MW instrument is superior to that of the IR instrument, particularly at lower altitudes. But MW instrument is not limited only to observations under clear sky conditions, unlike the IR instrument. The MW instrument can see down into clouds, and can disentangle the cloud and water vapour signals. This is a significant advantage.

Figure 9 shows the predicted temperature retrieval error. Again, note that the performance is slightly better than that of the IR instrument, and that we are not limited to clear-sky conditions. Also note that this performance is achieved WITHOUT employing detectors around the 50-60 $\mathrm{GHz} \mathrm{O} \mathrm{O}_{2}$ temperature sounding lines. As part of this study, further simulations will be run with the inclusion of these low frequency channels. We expect a large performance increase, particularly for temperature retrieval. The error increases at higher altitudes due to the absorption lines becoming narrower, as a result of decreased pressure broadening.

Figure 10 presents the predicted vertical resolution for water vapour retrieval. For the MW instrument, we achieve vertical resolution in the troposphere of better that $1.5 \mathrm{~km}$. This is a very large advantage over the microwave $\&$ sub-mm instruments under development for MetOp-SG (MWS, MWI, ICI). These instruments have very poor vertical resolution, typically "resolving" the atmosphere into $\sim 3$ layers. 
IR

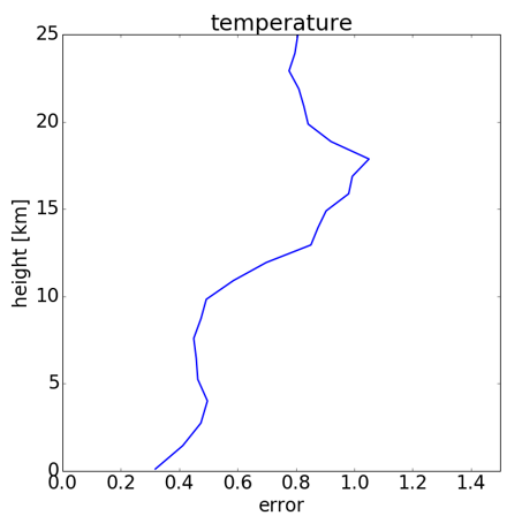

KID Microwave

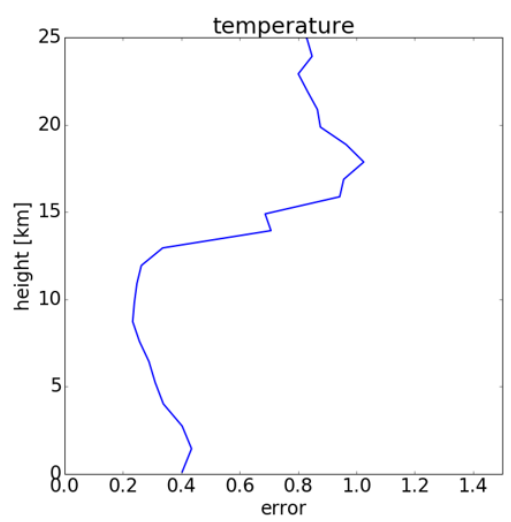

Figure 9. Predicted temperature retrieval error for IR and KID-based microwave instruments. The error shown is the absolute error in Kelvin.

\section{IR}

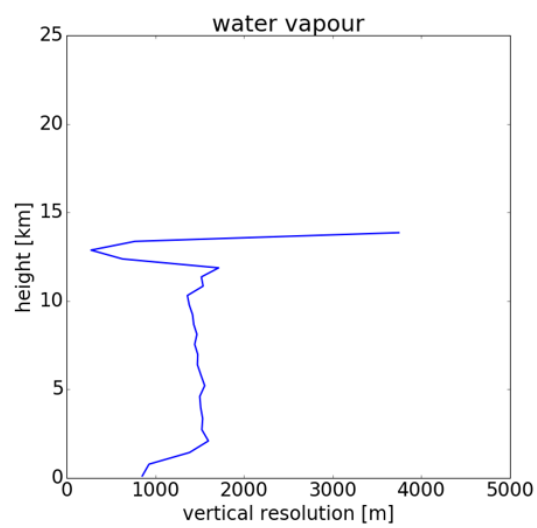

KID Microwave

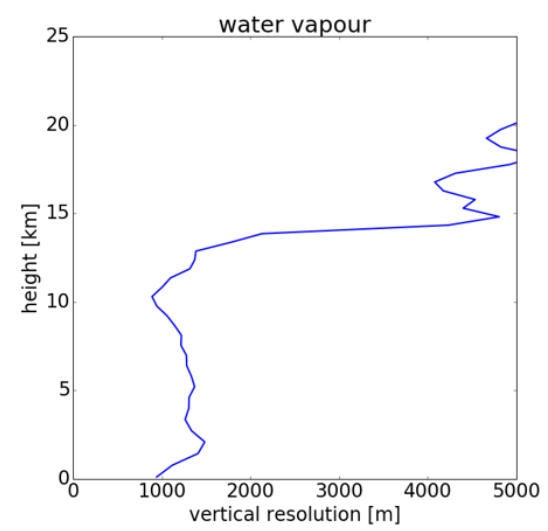

Figure 10. Predicted vertical resolution for water vapour retrieval. KID instrument vertical resolution in troposphere is better than $1.5 \mathrm{~km}$.

\section{CONCLUSIONS}

We have presented a new instrument concept for meteorology and climatology applications enabled by the use of novel on-chip superconducting filterbank spectrometers, coupled to transition-edge superconducting sensors. Such an instrument should significantly enhance weather forecasting ability, and reduce uncertainties in global circulation and climate models.

\section{ACKNOWLEDGEMENT}

We thank the UK Centre for Earth Observation and Space Technology (CEOI-ST), and the UK Science and Technology Facilities Council (STFC) for supporting this work.

\section{REFERENCES}

1. Rosenkranz, P.W., Retrieval of temperature and moisture profiles from AMSU-A and AMSU-B measurements. Geoscience and Remote Sensing, IEEE Transactions on, 2001. 39(11): p. 2429-2435.

2. Zhu, Y. and R. Gelaro, Observation sensitivity calculations using the adjoint of the Gridpoint Statistical Interpolation (GSI) analysis system. Monthly Weather Review, 2008. 136(1): p. 335-351.

3. Cardinali, C., Monitoring the observation impact on the short-range forecast. Quarterly Journal of the Royal Meteorological Society, 2009. 135(638): p. 239-250. 
4. Collard, A. and A. McNally, The assimilation of infrared atmospheric sounding interferometer radiances at ECMWF. Quarterly Journal of the Royal Meteorological Society, 2009. 135(641): p. 1044-1058.

5. Radnoti, G., et al., ECMWF Study on the Impact of Future Developments of the Space-based Observing System on Numerical Weather Precition. 2010: European Centre for Medium-Range Weather Forecasts.

6. McNally, T., Observing System Experiments to assess the impact of possible future degradation of the global satellite observing network. 2012: European Centre for Medium-Range Weather Forecasts.

7. English, S., et al., Impact of satellite data. 2013, ECMWF Technical Memorandum.

8. Lorenc, A.C. and R.T. Marriott, Forecast sensitivity to observations in the Met Office Global numerical weather prediction system. Quarterly Journal of the Royal Meteorological Society, 2014. 140(678): p. 209-224.

9. Hilton, F., et al., Hyperspectral earth observation from IASI: five years of accomplishments, B. Am. Meteorol. Soc., 93, 347-370, doi: 10.1175. 2012, BAMS-D-11-00027.1.

10. Aires, F., et al., A new neural network approach including first-guess for retrieval of atmospheric water vapor, cloud liquid water path, surface temperature and emissivities over land from satellite microwave observations. 2000.

11. Karbou, F., É. Gérard, and F. Rabier, Microwave land emissivity and skin temperature for amsu- $A$ and-B assimilation over land. Quarterly Journal of the Royal Meteorological Society, 2006. 132(620): p. 2333-2355.

12. Karbou, F., et al., Global 4DVAR assimilation and forecast experiments using AMSU observations over land. Part II: Impacts of assimilating surface-sensitive channels on the African monsoon during AMMA. Weather and Forecasting, 2010. 25(1): p. 20-36.

13. Karbou, F., F. Rabier, and C. Prigent, The assimilation of observations from the Advanced Microwave Sounding Unit over sea ice in the French global numerical weather prediction system. Monthly Weather Review, 2014. 142(1): p. 125-140.

14. Aires, F., et al., Microwave hyperspectral measurements for temperature and humidity atmospheric profiling from satellite: The clear-sky case. Journal of Geophysical Research: Atmospheres, 2015. 120(21).

15. Mahfouf, J.F., et al., Information content on temperature and water vapour from a hyper-spectral microwave sensor. Quarterly Journal of the Royal Meteorological Society, 2015. 141(693): p. 3268-3284.

16. Prigent, C., et al., Final Report - Use of Spectral Information at Microwave Region for Numerical Weather Prediction, in Use of Spectral Information at Microwave Region for Numerical Weather Prediction. ESA contract number 4000105721/12/NL/AF. 2015.

17. Aires, F., Measure and exploitation of multisensor and multiwavelength synergy for remote sensing: 1. Theoretical considerations. Journal of Geophysical Research: Atmospheres, 2011. 116(D2).

18. Aires, F., et al., Measure and exploitation of multisensor and multiwavelength synergy for remote sensing: 2. Application to the retrieval of atmospheric temperature and water vapor from MetOp. Journal of Geophysical Research: Atmospheres, 2011. 116(D2).

19. Stocker, T., et al., IPCC Third Assessment Report-Climate Change 2001: The Scientific Basis, chap. Physical climate processes and feedbacks. WMO, 2001.

20. Doms, G. and U. Schättler, The nonhydrostatic limited-area model LM (Lokal-Modell) of DWD. Part I: Scientific documentation, Deutscher Wetterdienst (DWD), 1999.

21. Roeckner, E., et al., The atmospheric general circulation model ECHAM 5. PART I: Model description. 2003.

22. Lohmann, U. and E. Roeckner, Design and performance of a new cloud microphysics scheme developed for the ECHAM general circulation model. Climate Dynamics, 1996. 12(8): p. 557-572.

23. Wilson, D., The impact of a physically based microphysical scheme on the climate simulation of the Meteorological Office Unified Model. Quarterly Journal of the Royal Meteorological Society, 2000. 126(565): p. 1281-1300.

24. Reinhardt, T. and U. Wacker, Impact of ice particle habits on simulated clouds. Geophysical research letters, 2004. 31(21).

25. Heymsfield, A.J. and J. Iaquinta, Cirrus crystal terminal velocities. Journal of the atmospheric sciences, 2000. 57(7): p. 916-938.

26. Kunzi, K., CIWSIR Mission Proposal. Proposal to ESA Earth Explorer Opportunity Mission Call, 2001.

27. Evans, K.F., et al., Modeling of submillimeter passive remote sensing of cirrus clouds. Journal of Applied Meteorology, 1998. 37(2): p. 184-205.

28. John, V. and B. Soden, Does convectively-detrained cloud ice enhance water vapor feedback? Geophysical research letters, 2006. $\mathbf{3 3}(20)$. 
29. Jarret, M., et al., Establishment of mission and instrument requirements to observe cirrus clouds at submillimetre wavelengths. Final Report, ESTEC contract, 2007(19053/05).

30. Endo, A., et al. Development of DESHIMA: a redshift machine based on a superconducting on-chip filterbank. in SPIE Astronomical Telescopes + Instrumentation. 2012. International Society for Optics and Photonics.

31. Shirokoff, E., et al., Design and Performance of SuperSpec: An On-Chip, KID-Based, mm-Wavelength Spectrometer. Journal of Low Temperature Physics, 2014. 176(5-6): p. 657-662.

32. Griffin, M., et al. SPACEKIDS: kinetic inductance detectors for space applications. in SPIE Astronomical Telescopes + Instrumentation. 2016. International Society for Optics and Photonics.

33. Goldie, D., et al., Ultra-low-noise MoCu transition edge sensors for space applications. Journal of Applied Physics, 2011. 109(8): p. 084507.

34. Rostem, K., et al. Thermal conductance measurements for the development of ultra low-noise transition-edge sensors with a new method for measuring the noise equivalent power. in SPIE Astronomical Telescopes+ Instrumentation. 2008. International Society for Optics and Photonics.

35. Glowacka, D., et al., A fabrication process for microstrip-coupled superconducting transition edge sensors giving highly reproducible device characteristics. Journal of Low Temperature Physics, 2008. 151(1): p. 249254.

36. Goldie, D., et al., Transition edge sensors for bolometric applications: responsivity and saturation. Journal of Applied Physics, 2008. 103(8): p. 084509.

37. Mauskopf, P., et al. Development of transition edge superconducting bolometers for the SAFARI Far-Infrared spectrometer on the SPICA space-borne telescope. in SPIE Astronomical Telescopes+ Instrumentation. 2008. International Society for Optics and Photonics.

38. Goldie, D., et al. Ultra-low-noise transition edge sensors for the SAFARI L-band on SPICA. in SPIE Astronomical Telescopes+ Instrumentation. 2012. International Society for Optics and Photonics.

39. Jackson, B., et al., The SPICA-SAFARI detector system: TES detector arrays with frequency-division multiplexed SQUID readout. IEEE Transactions on Terahertz Science and Technology, 2012. 2(1): p. 12-21.

40. van Weers, H., et al. TES-detector based focal plane assembly key-technology developments for ATHENA and SAFARI. in SPIE Astronomical Telescopes+ Instrumentation. 2014. International Society for Optics and Photonics.

41. Osman, D., et al., Transition edge sensors with few-mode ballistic thermal isolation. Journal of Applied Physics, 2014. 116(6): p. 064506.

42. D'Addio, S., et al. The microwave radiometers on-board MetOp second generation satellites. in Metrology for Aerospace (MetroAeroSpace), 2014 IEEE. 2014. IEEE.

43. Varchetta, S., et al. Main design parameters and imaging/radiometric performances of the METOP Second Generation conically scanning radiometers. in Microwave Radiometry and Remote Sensing of the Environment (MicroRad), 2012 12th Specialist Meeting on. 2012. IEEE.

44. Alberti, G., et al. TWO Microwave Imaging radiometers for MetOp Second Generation. in Advances in Radar and Remote Sensing (TyWRRS), 2012 Tyrrhenian Workshop on. 2012.

45. Kangas, V., et al. METOP Second Generation Microwave Sounding And Microwave Imaging Missions. in 2012 EUMETSAT Meteorological Satellite Conference. 2012.

46. Poglitsch, A., et al., The photodetector array camera and spectrometer (PACS) on the Herschel space observatory. Astronomy \& astrophysics, 2010. 518: p. L2.

47. Griffin, M.J., et al., The Herschel-SPIRE instrument and its in-flight performance. Astronomy and Astrophysics, 2010. 518: p. L3.

48. Tauber, J.A., et al., Planck pre-launch status: The Planck mission. Astronomy \& Astrophysics, 2010. 520: p. A1.

49. Buehler, S., et al., ARTS, the atmospheric radiative transfer simulator. Journal of Quantitative Spectroscopy and Radiative Transfer, 2005. 91(1): p. 65-93. 